\title{
Combination of apodized pupil and phase mask coronagraph for SCExAO at Subaru Telescope
}

Nishikawa, Jun, Murakami, Naoshi, Lozi, Julien, Guyon, Olivier, Habu, Keiichiro, et al.

Jun Nishikawa, Naoshi Murakami, Julien Lozi, Olivier Guyon, Keiichiro Habu, Hikaru Ichien, Kenta Yoneta, Seiji Sudoh, Kosuke Kumaki, Shiomi Kumagai, Nemanja Jovanovic, Frantz Martinache, "Combination of apodized pupil and phase mask coronagraph for SCEXAO at Subaru Telescope," Proc. SPIE 11447, Ground-based and Airborne Instrumentation for Astronomy VIII, 114474T (13 December 2020); doi: 10.1117/12.2560839 


\title{
Combination of apodized pupil and phase mask coronagraph for SCExAO at Subaru Telescope
}

\author{
Jun Nishikawa*abc, Naoshi Murakamid ${ }^{\mathrm{d}}$, Julien Lozi ${ }^{\mathrm{e}}$, Olivier Guyon ${ }^{\mathrm{efgc}}$, Keiichiro Habu ${ }^{\mathrm{d}}$, Hikaru \\ Ichien $^{\mathrm{d}}$, Kenta Yoneta ${ }^{\mathrm{d}}$, Seiji Sudo ${ }^{\mathrm{d}}$, Kosuke Kumaki ${ }^{\mathrm{h}, \mathrm{a}}$, Shiomi Kumagaih ${ }^{\mathrm{h}}$, Nemanja Jovanovic ${ }^{\mathrm{i}}$, \\ Frantz Martinache $\mathrm{e}^{\mathrm{j}}$ \\ ${ }^{a}$ National Astronomical Observatory of Japan, 2-21-1 Osawa, Mitaka, Tokyo, Japan 181-8588; \\ ${ }^{\mathrm{b}}$ Graduate University for Advanced Studies, 2-21-1 Osawa, Mitaka, Tokyo, Japan 181-8588; \\ ${ }^{\mathrm{c} A s t r o b i o l g y ~ C e n t e r, ~ N I N S, ~ 2-21-1 ~ O s a w a, ~ M i t a k a, ~ T o k y o, ~ J a p a n ~ 181-8588 ; ~}{ }^{\mathrm{d}}$ Faculty of Engineering, \\ Hokkaido University, Kita-13, Nishi-8, Kita-ku, Sapporo, Hokkaido, Japan 060-8628; ${ }^{\text {eSubaru }}$ \\ Telescope, NAOJ, Hilo, Hawaii, USA 96720; 'Steward Observatory, University of Arizona, Tucson, \\ AZ, 85721, U.S.A.; ${ }^{\mathrm{g} C}$ College of Optical Sciences, University of Arizona, Tucson, AZ 85721, U.S.A.; \\ ${ }^{h}$ Faculty of Science and Engineering, Nihon University, 1-8 Kandasurugadai, Chiyoda-ku, Japan \\ 101-0062; i California Institute of Technology, 1200 E California Blvd, Pasadena, CA 91125, U.S.A; \\ ${ }^{\mathrm{j}}$ Laboratoire Lagrange, Université Côte d'Azur, Observatoire de la Côte d'Azur, CNRS, Parc \\ Valrose, Bât. H. FIZEAU, 06108 Nice, France
}

\begin{abstract}
Subaru telescope has been operating a high-contrast imaging instruments called Subaru coronagraphic extreme adaptive optics (SCExAO) which is used for exoplanet research. We are developing phase mask coronagraphs using photonic crystal wave plates inside the SCExAO. An eight-octant phase mask (8OPM) of three-layer achromatic structure has been fabricated as a second generation. It was designed for $\mathrm{J}$ and $\mathrm{H}$ band to reach $10^{-5}$ contrast, and $\mathrm{Ks}$ band to $10^{-4}$. A retardation and a coronagraphic performance of the 8OPM were confirmed almost as designed at $1550 \mathrm{~nm}$. An apodised (binary shaped) pupil to be used with the 8OPM was also studied to suppress diffracted light by the secondary shadow and spiders. We confirmed a performance of the combination of the apodizer and the 8OPM at visible wavelengths in a lab. We optimized the apodizer for a pupil of the SCExAO where we obtained a transmission of $50 \%$ and a contrast of $10^{-4}$ at the center and $10^{-6}$ at outer region. We manufactured the designed apodizer to be installed in SCExAO for infrared observations.
\end{abstract}

Keywords: exoplanet, coronagraph, adaptive optics, phase mask, apodizer, shaped pupil

\section{INTRODUCTION}

One of the most interesting themes in modern astronomy is to characterize exoplanets and investigate biosignatures in their spectrums by detecting reflected light with direct imaging techniques ${ }^{1}$. In order to detect the reflected light high contrast imaging instruments with $10^{-8}$ to $10^{-10}$ are required, which will be achieved by future large telescopes in space and at the ground. A high contrast imaging instrument consists of an adaptive optics system to reduce speckle noise by compensating wavefront errors and a coronagraph which suppresses diffracted light of the telescope optics. The Lyot's coronagraph to observe solar corona applied an opaque occulting disk to hide the Sun at a focus of a telescope and the Lyot's stop to cut diffracted light at a re-imaged pupil. The coronagraph optics produced an image of the faint corona at the final focus successfully. To observe very faint exoplanets around stars, many kinds of coronagraph styles have been developed, a nulling interferometer at a pupil plane, apodization at the pupil, a phase control at a focal plane, and an intensity modulation at the focal plane. We are developing phase mask coronagraphs at the focal plane, and that in combination with apodized pupils. In this paper we describe mainly an eight-octant phase mask (8OPM) in the infrared to equip in Subaru telescope and the shaped pupil to be used together.

*jun.nishikawa@nao.ac.jp; phone 81422 34-3612;

Ground-based and Airborne Instrumentation for Astronomy VIII, edited by Christopher J. Evans,

Julia J. Bryant, Kentaro Motohara, Proc. of SPIE Vol. 11447, 114474T · C 2020

SPIE $\cdot$ CCC code: $0277-786 \mathrm{X} / 20 / \$ 21 \cdot$ doi: $10.1117 / 12.2560839$ 


\section{PHOTONIC CRYSTAL PHASE MASK CORONAGRAPHS}

\subsection{Photonic crystal phase mask coronagraphs}

We have developed phase mask coronagraphs using photonic crystal wave plate with very fine structures of sub-um accuracy $^{2}$ :

- concentric pattern (2nd order vortex)

- 4-quadrant phase mask (4QPM, $0 / \pi$ digitized 2 nd-order vortex)

- 8-octant phase mask (8OPM, $0 / \pi$ digitized 4 th-order vortex)

- 32-segment 4th order vortex.

The 2nd-order vortex and the 8OPM were tested at HCIT(JPL) and THD(Paris) reaching to several of $10^{-8}$ contrast. An H-band 8OPM has been already installed in Subaru coronagraphic extreme adaptive optics (SCExAO) ${ }^{3}$.
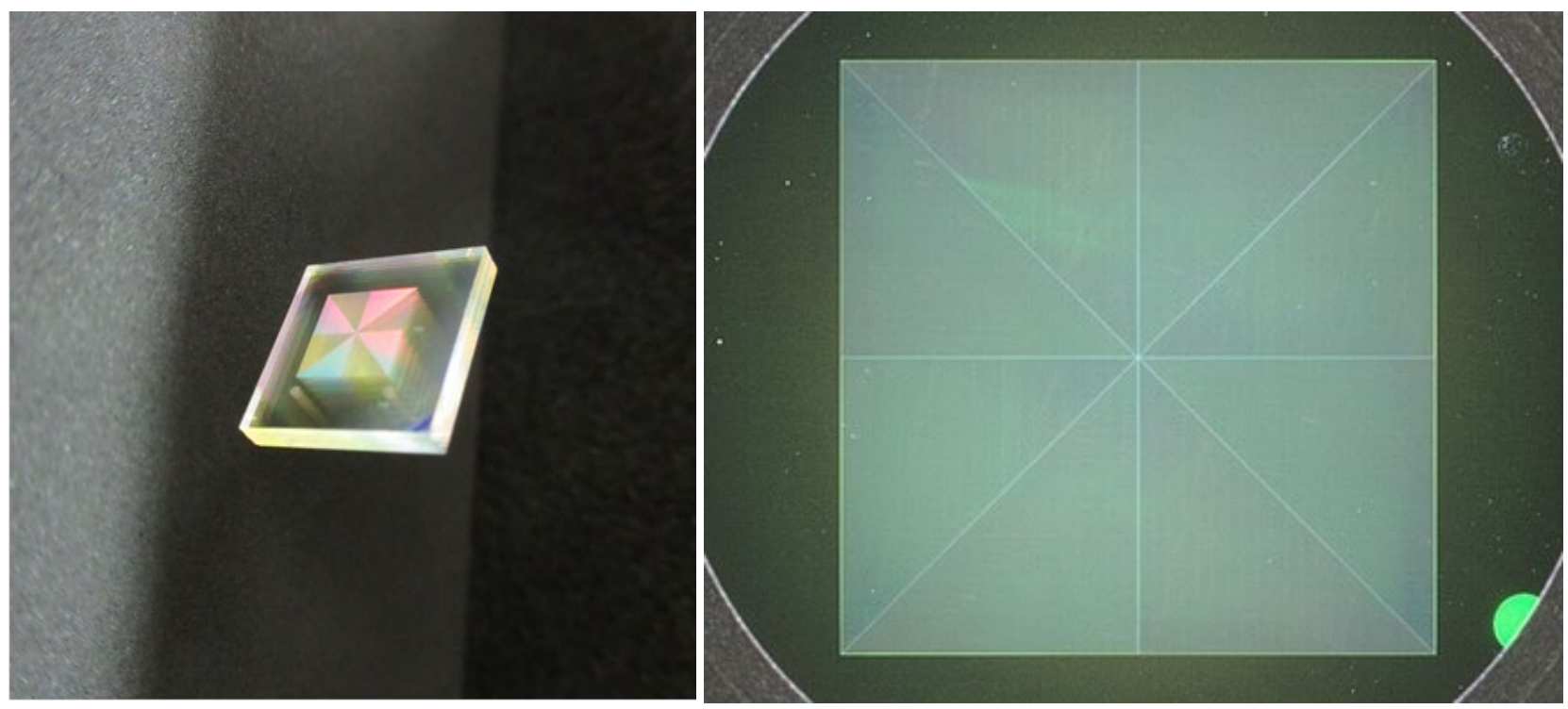

Figure 1.Three-layer IR 8OPM optimized for J,H and Ks band just after gluing.

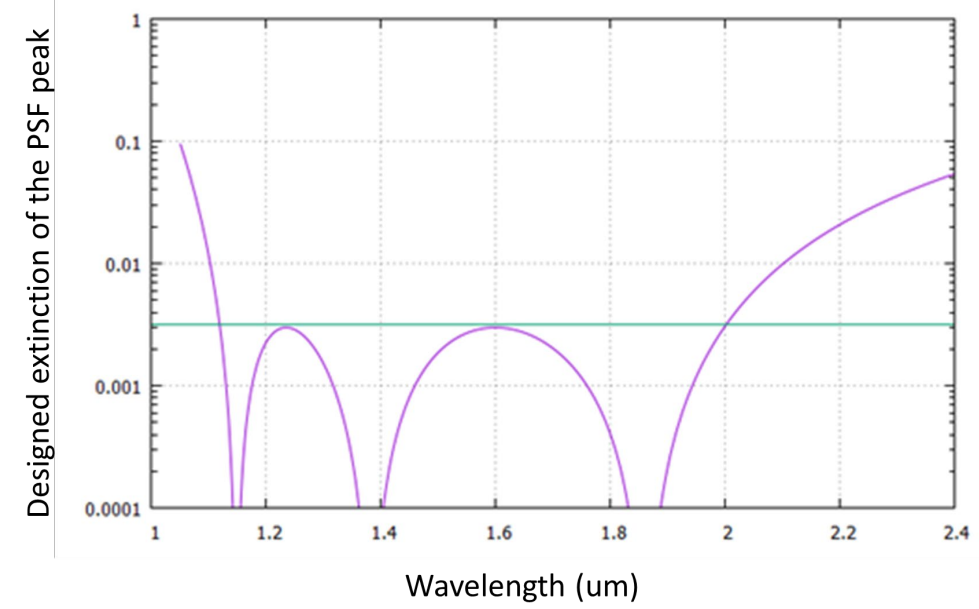

Figure 2. Designed extinction ratio of the PSF peak for the three-layer IR 8OPM in J,H and K band.

Recently we manufactured a three-layer IR 8OPM. It was designed as achromatic at the J, $\mathrm{H}$ and Ks band in the infrared. The tree layers have designed orientations of their fast axes as the wave plates to apply Pancharatnam phase ${ }^{4}$. Each layer 
was once fabricated in a substrate, cut to separate each other, and glued into one optical element, as shown in left panel of Fig. 1. It was mounted in a holder as shown in right panel of Fig. 1 to install at the filter wheel of the SCExAO.

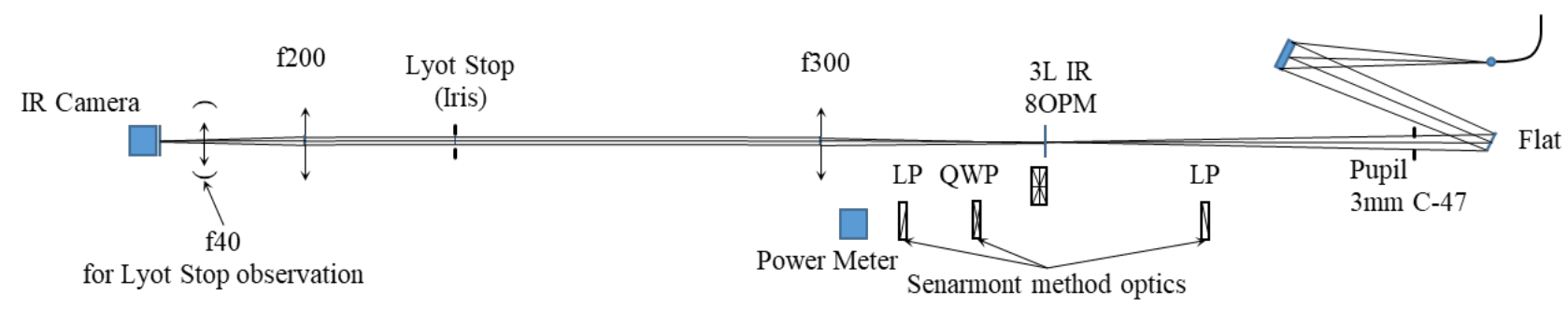

Figure 3. Coronagraph optics to see the extinction performance of the three-layer IR 8OPM.

The extinction ratio at the PSF peak in the achromatic design was about $3 \times 10^{-3}$ between about $1.1 \mathrm{um}$ and 2.0 um and better than $3 \times 10^{-4}$ up to 2.2 um as shown in Fig. 2, and then achievable contrasts at halo region would be expected $1 \times 10^{-5}$ at $\mathrm{J}$ and $\mathrm{H}$ bands and $1 \times 10^{-4}$ at Ks band.

We constructed a coronagraph optics as shown in Fig. 3 and obtained an image at the Lyot plane with the three-layer IR 8OPM shown in Fig. 4 which indicated a good extinction within the re-imaged pupil at the Lyot plane. An extinction ratio of the PSF peak at the final focal plane was about $10^{-3}$ at $1550 \mathrm{~nm}$ which was almost coincident to its achromatic design.

We also measured a retardation of the three-layer IR 8OPM by inserting Senarmont method optics and a power meter to the optics shown in Fig, 3. We found that the retardation of a segment of the mask was about 185 degrees at $1550 \mathrm{~nm}$, where the error was about 4 degrees. We expect that the half-wave retardation will be proved as designed when the peak contrast at several wavelengths are confirmed by an artificial light experiment in SCExAO.

Figure 4. Lyot plane image of the three-layer IR 8OPM at 1550nm. 

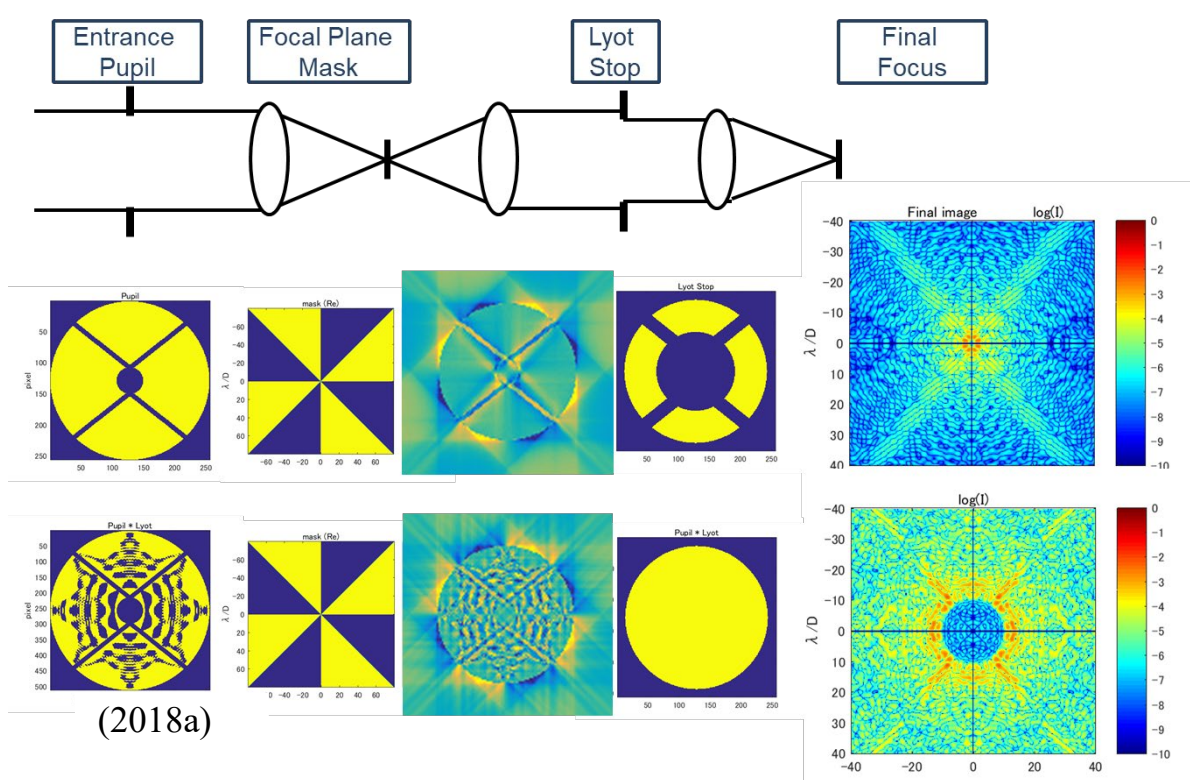

Figure 5. Principle of 8OPM with apodized pupil.

\section{8OPM WITH APODIZED PUPIL}

Most of coronagraphs work well with a circular aperture, but bright diffracted light remains at a final focus if the aperture is obstructed by a secondary and spiders. A lens pair which fills up the secondary shadow has been also installed in SCExAO. It is, however, not easy to manufacture and set the lens pair with very low aberrations ${ }^{5}$. We are going to introduce an apodized (binary) pupil combining with the 8OPM to reduce the diffracted light instead of the lens pair. Instead an optimized apodizer at a pupil combined with the focal plane phase mask can suppress the diffracted light within a region of interest at the focal plane for exoplanet search ${ }^{6}$, that principal is shown in Fig.5.

Firstly we manufactured an apodizer shown in Fig. 6 which had the Subaru-like pattern indicated as (2018a) in Fig. 5 with a pupil diameter of $2.5 \mathrm{~mm}$ and made optical experiments. We got a contrast about $1 \times 10^{-5}$ between 0 to 10 lambda/D which was the same as that with a circular aperture as shown in Fig 6.
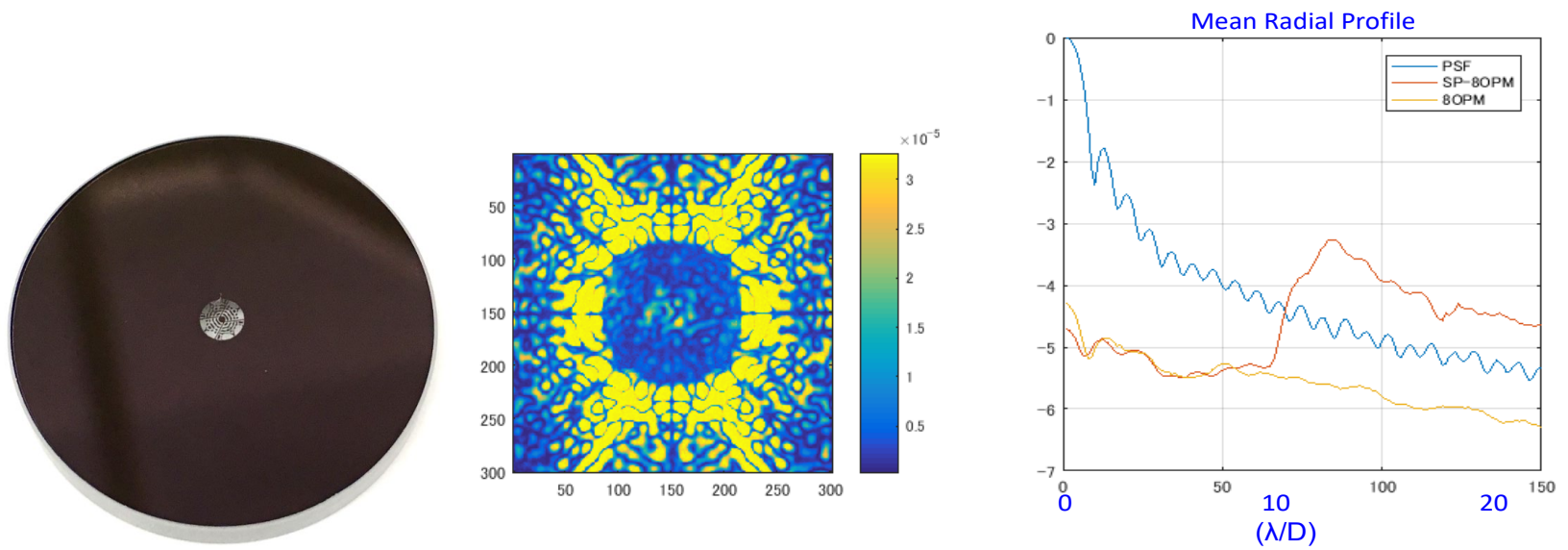

Figure 6. Manufactured test apodizer and its performance. 

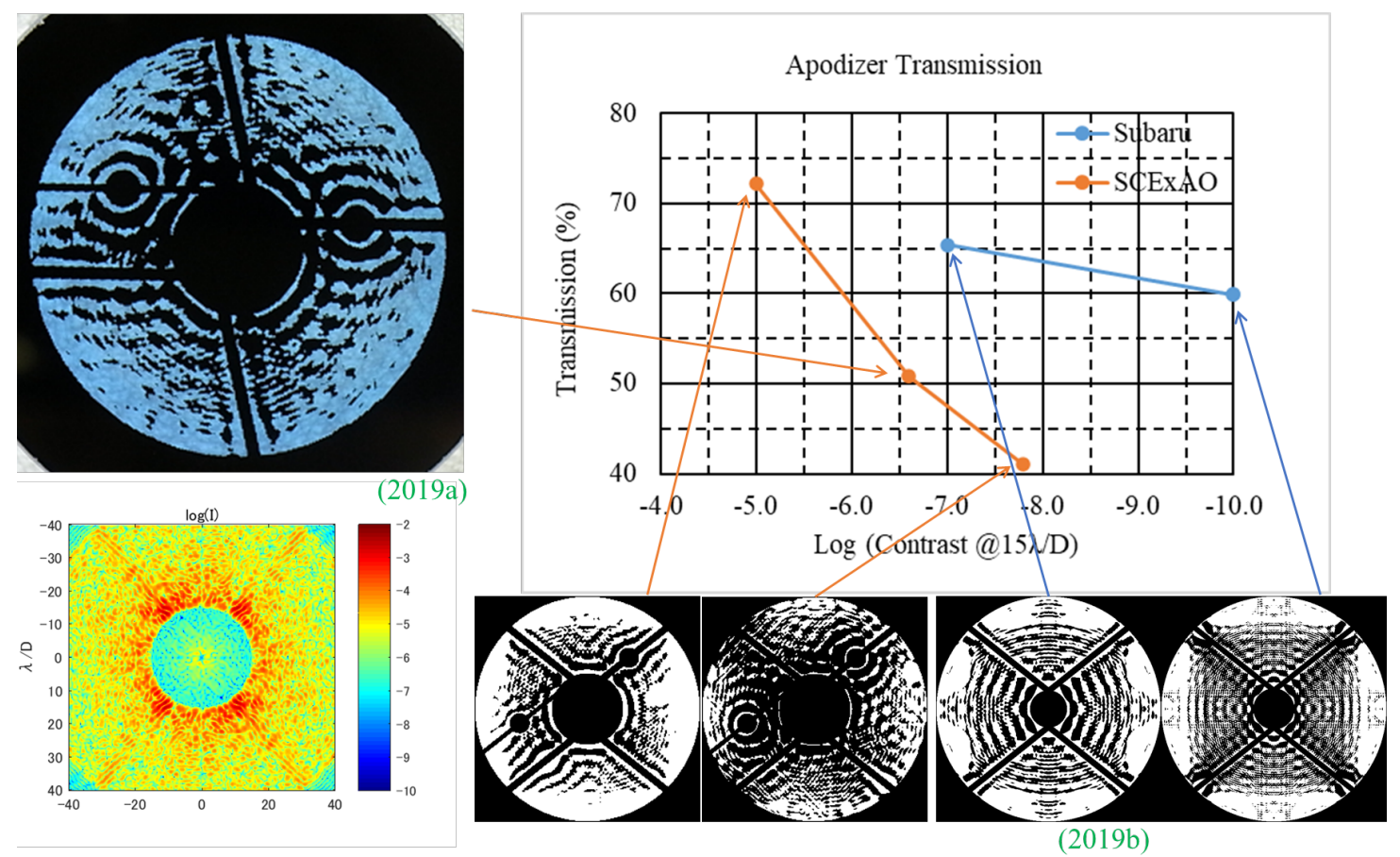

Figure 7. (upper left) Fabricated apodizer for SCExAO, (lower left) expected contrast, (right) apodizer transmission.

Transmission of the apodizer depends on the target contrast. We estimated the transmission in two cases as shown right panels in Fig.7. One is for the SCExAO and another is for the Subaru-like pupil shape with a smaller obstruction. We found that the dependence became hard if the original obstruction was large. We adopted the solution with the transmission of $50 \%$ (2019a) for SCExAO with a designed contrast of $3 \times 10^{-7}$ at $15 \mathrm{lambda} / \mathrm{D}$ and $3 \times 10^{-5}$ at 1 lambda/D as shown at lower left in Fig 7. A manufactured apodizer was shown at upper left in Fig 7 which pupil diameter was 17.4 $\mathrm{mm}$. Apodized pupil with $\mathrm{T}=60 \%$ (2019b) was also fabricated for optical experiments.

Other pupil optimization with the 8OPM (except WSOUV and SCExAO) are shown in Fig. 8 where the radius of the focal target region was set to 9 lambda/D. These solutions indicated a possibility of a high contrast by the combination of the 8OPM and apodizer. We tried the WFIRST (Roman) pupil in 2016 and obtained a design of $10^{-12}$ at an on-axis point source but it was difficult to keep the contrast for an off-axis ray of 1 mas where the leak intensity was about $10^{-7}$. We need to make further investigation taking care of this point to apply for very high-contrast space telescopes, although it would be less problem for ground-based telescopes. At the rightmost of Fig. 8, a 6th-order vortex mask with 48-segment was applied to the SCExAO pupil for future studies. The focal plane mask with segments more than 8 has a calculation error at the mask operation in the focal plane, which must be considered carefully in the design process of the apodizers.

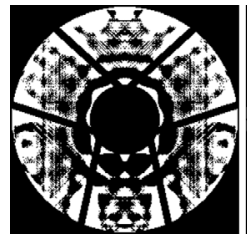

WFIRST

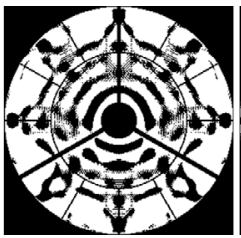

OST

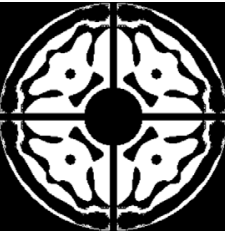

WSOUV (no mask)

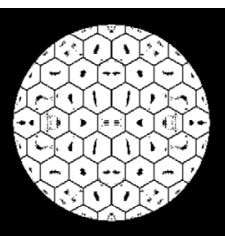

LUVOIR-B

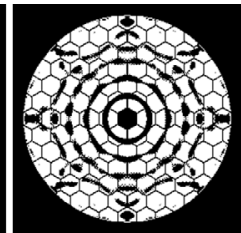

LUVOIR-A

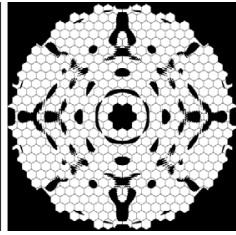

TMT

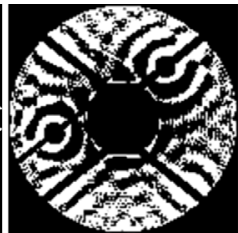

SCExAO (6-order vortex)

Figure 8. Apodizer design examples for other telescopes. 


\section{CONCLUSION}

We manufactured a three-layer 8-octant phase mask by a photonic crystal waveplate which was achromatic for J, H, and Ks band. The mask will be evaluated in SCExAO. An apodizer to be combined with the 8OPM was designed and fabricated, which would suppress the diffracted light of the secondary shadow and spiders down to $1 \times 10^{-4}$ at the center and $1 \times 10^{-6}$ at $15 \mathrm{lambda} / \mathrm{D}$. The combination performance will be also evaluated in SCExAO. We need to make further studies for the combination of apodizers and phase masks on the off-axis ray and the higher-order vortex masks.

\section{ACKNOWLEDGEMENT}

This work was supported partly by JSPS KAKENHI (Grant Number 19H01932) and Astrobiology Center Project (AB301009). Experiments were made at a lab in the Advanced Technology Center of the NAOJ.

\section{REFERENCES}

[1] Gaudi, B. S. et al., "The Habitable Exoplanet Observatory (HabEx) Mission Concept Study Final Report," arXiv:2001.06683v2 (2020).

[2] Murakami, N., Nishikawa, J., Tamura, M., Serabyn, E., Traub, W. A., Liewer, K. M., Moody, D. C., Trauger, J. T., Guyon, O., Martinache, F., Jovanovic, N., Singh, G., Oshiyama, F., Shoji, H., Sakamoto, M., Hamaguchi, S., Oka, K., and Baba N., "Recent progress on phase-mask coronagraphy based on photonic-crystal technology, Proc. SPIE 9143, 914334 (2014).

[3] Jovanovic, N., Martinache, F., Guyon, O., Clergeon, C., Singh, G., Kudo, T., Garrel, V., Newman, K., Doughty, D., Lozi, J., Males, J., Minowa, Y., Hayano, Y., Takato, N., Morino, J., Kuhn, J., Serabyn, E., Norris, B., Tuthill, P., Schworer, G. Stewart, P., Close, L., Huby, E., Perrin, G., Lacour, S., Gauchet, L., Vievard, S., Murakami, N., Oshiyama, F., Baba, N., Matsuo, T., Nishikawa, J., Tamura, M., Lai, O., Marchis, F., Duchene, G., Kotani, T., Woillez, J., "The Subaru Coronagraphic Extreme Adaptive Optics System: Enabling High-Contrast Imaging on Solar-System Scales," PASP 127, 890-910 (2015)

[4] Murakami, N., Nishikawa, J., Akaiwa, N., Komuro, Y., Baba, N. and Tamura, M., "A three-layer eight-octant phase mask towards broadband high-contrast observations," Proc. SPIE 9912, 99126G (2016).

[5] Oshiyama, F., Murakami, N., Guyon, O., Martinache, F., Baba, N., Matsuo, T., Nishikawa, J., Tamura, M. "Central-Obscuration Removal Plates for Focal-Plane Phase-Mask Coronagraphs with a Centrally-Obscured Telescope," PASP, 126:270-279 (2014).

[6] Carlotti, A., "Apodized phase mask coronagraphs for arbitrary apertures,” A\&A 551, A10 (2013). 